become a new weapon for the oncologist's armamentarium. An agent like BIM SAHB that targets the full panoply of prosurvival guardians, and is expected to also promote BAX activation directly, might have been expected to decimate many normal tissues. That was not observed under conditions in which a substantial proportion of tumor cells were killed. Presumably, the malignant cells are more sensitive than many normal cells, because the stresses induced by their disturbed cell cycle checkpoints have rendered them primed for apoptosis (13), perhaps by increasing their level of BIM. Thus, the results of the work by LaBelle et al. suggest that there may well be a therapeutic window for peptides like the BIM SAHB in treatment of refractory leukemias.

\section{Acknowledgments}

This work was supported by the National Health and Medical Research Council (NHMRC), Australia (program grant 461221 and fellowship 516703), and was facilitated by Victorian State Government Operational Infrastructure Support and Australian Government NHMRC IRIISS.

Address for correspondence: Jerry M. Adams, Walter and Eliza Hall Institute of Medical Research, 1G Royal Parade, Parkville 3052, Victoria, Australia. Phone: 61.3. 9345.2491; Fax: 61.3.9347.0852; E-mail: adams@wehi.edu.au.

1. Strasser A, Cory S, Adams JM. Deciphering the rules of programmed cell death to improve therapy of cancer and other diseases. EMBOJ. 2011;30(18):3667-3683.

2. Lessene G, Czabotar PE, Colman PM. BCL-2 family antagonists for cancer therapy. Nat Rev Drug Discov. 2008;7(12):989-1000

3. Oltersdorf T, et al. An inhibitor of Bcl-2 family proteins induces regression of solid tumours. Nature. 2005;435(7042):677-681

4. Tse C, et al. ABT-263: a potent and orally bioavailable Bcl-2 family inhibitor. Cancer Res. 2008; 68(9):3421-3428.

5. van Delft MF, et al. The BH3 mimetic ABT-737 targets selective $\mathrm{Bcl}-2$ proteins and efficiently induces apoptosis via Bak/Bax if Mcl-1 is neutralized. Cancer Cell. 2006;10(5):389-399.

6. Roberts AW, et al. Substantial susceptibility of chronic lymphocytic leukemia to BCL2 inhibition: results of a phase I study of navitoclax in patients with relapsed or refractory disease.J Clin Oncol. 2012; 30(5):488-496.

7. Mason KD, et al. Programmed anuclear cell death delimits platelet life span. Cell. 2007; 128(6):1173-1186

8. LaBelle JL, et al. A stapled BIM peptide overcomes apoptotic resistance in hematologic cancers. J Clin Invest. 2012;122(6):2018-2031.

9. Walensky LD, et al. Activation of apoptosis in vivo by a hydrocarbon-stapled BH3 helix. Science. 2004;305(5689):1466-1470.

10. Gavathiotis E, et al. BAX activation is initiated at a novel interaction site. Nature. 2008; 455(7216):1076-1081.

11. Gavathiotis E, Reyna DE, Davis ML, Bird GH, Walensky LD. BH3-triggered structural reorganization drives the activation of proapoptotic BAX. Mol Cell. 2010;40(3):481-492.

12. Chen L, et al. Differential targeting of pro-survival $\mathrm{Bcl}-2$ proteins by their $\mathrm{BH} 3$-only ligands allows complementary apoptotic function. Mol Cell. 2005;17(3):393-403.

13. Certo $M$, et al. Mitochondria primed by death signals determine cellular addiction to antiapoptotic BCL-2 family members. Cancer Cell. 2006;9(5):351-365.

14. Bouillet $\mathrm{P}$, et al. Proapoptotic $\mathrm{Bcl}-2$ relative Bim required for certain apoptotic responses, leukocyte homeostasis, and to preclude autoimmunity. Science. 1999;286(5445):1735-1738.

\title{
Taming endothelial activation with a microRNA
}

\author{
Jason E. Fish and Myron I. Cybulsky
}

\begin{abstract}
Division of Cellular and Molecular Biology, Toronto General Research Institute, University Health Network, Toronto, Ontario, Canada. Department of Laboratory Medicine and Pathobiology, University of Toronto, Toronto, Ontario, Canada. Heart and Stroke Richard Lewar Centre of Excellence in Cardiovascular Research, Toronto, Ontario, Canada.
\end{abstract}

\begin{abstract}
Inflammation plays an essential role in vascular pathologies, including those associated with sepsis and atherosclerosis. Identifying negative regulators of inflammatory signaling pathways may provide novel therapeutic targets for these diseases. In this issue of the JCI, Sun et al. show that in endothelial cells, microRNA-181b (miR-181b) plays a vital role in controlling inflammation by targeting importin- $\alpha 3$, a regulator of NF- $\kappa B$ nuclear import. These findings provide compelling evidence that modulation of microRNAs may be a useful therapeutic approach for inflammatory vascular diseases.
\end{abstract}

\section{Vascular endothelium and inflammation}

The vascular endothelium forms the interface between blood and tissues and plays a critical and active role in maintaining blood vessel and tissue homeostasis as well as a nonthrombotic and nonadhesive vascular surface. Endothelial cell activation by proinflammatory stimuli promotes leukocyte recruitment from the blood into extravascular tissues and thus contributes to the

Conflict of interest: The authors have declared that no conflict of interest exists.

Citation for this article: J Clin Invest. 2012; 122(6):1967-1970. doi:10.1172/JCI63818. pathogenesis of various inflammatory diseases (1). Multiple signaling pathways participate in this process, but the NF-кB signaling pathway plays a particularly central role. NF- $\mathrm{KB}$ signal transduction in endothelium culminates in the expression of multiple proinflammatory genes, including cell adhesion molecules such as E- and P-selectin, vascular cell adhesion molecule 1 (VCAM-1), and intercellular adhesion molecule 1 (ICAM-1), as well as various chemokines and cytokines. Since endothelial cell activation contributes to the pathogenesis of multiple inflammatory and immune conditions, targeting NF- $\mathrm{KB}$ signal transduction is an attractive poten- tial therapeutic strategy. However, enthusiasm should be tempered because a subset of NF-кB target genes promotes cell survival under conditions of stress. It is also important to target the "right" cells because inhibition of NF- $\mathrm{KB}$ signaling in endothelial cells reduces atherosclerosis, whereas inhibition in macrophages promotes cell death and exacerbates atherosclerosis $(2,3)$. In this issue of the JCI, Sun et al. (4) explore a novel microRNA-based approach to regulating NF- $\mathrm{KB}$ signaling and provide insights into its therapeutic utility.

\section{NF-KB signal transduction}

The NF- $\mathrm{B} /$ Rel family consists of protein homo- and heterodimers, each with different DNA binding and activation specificity (5). The $\mathrm{p} 50 / \mathrm{p} 65$ heterodimer is the prototypic NF- $\kappa \mathrm{B}$ and is predominant in endothelial cells. In quiescent cells, NF-кB is predominantly localized in the cytoplasm, where it is retained through association with an inhibitor protein (IкB) (ref. 5 and Figure 1). There are several IкBs, and 


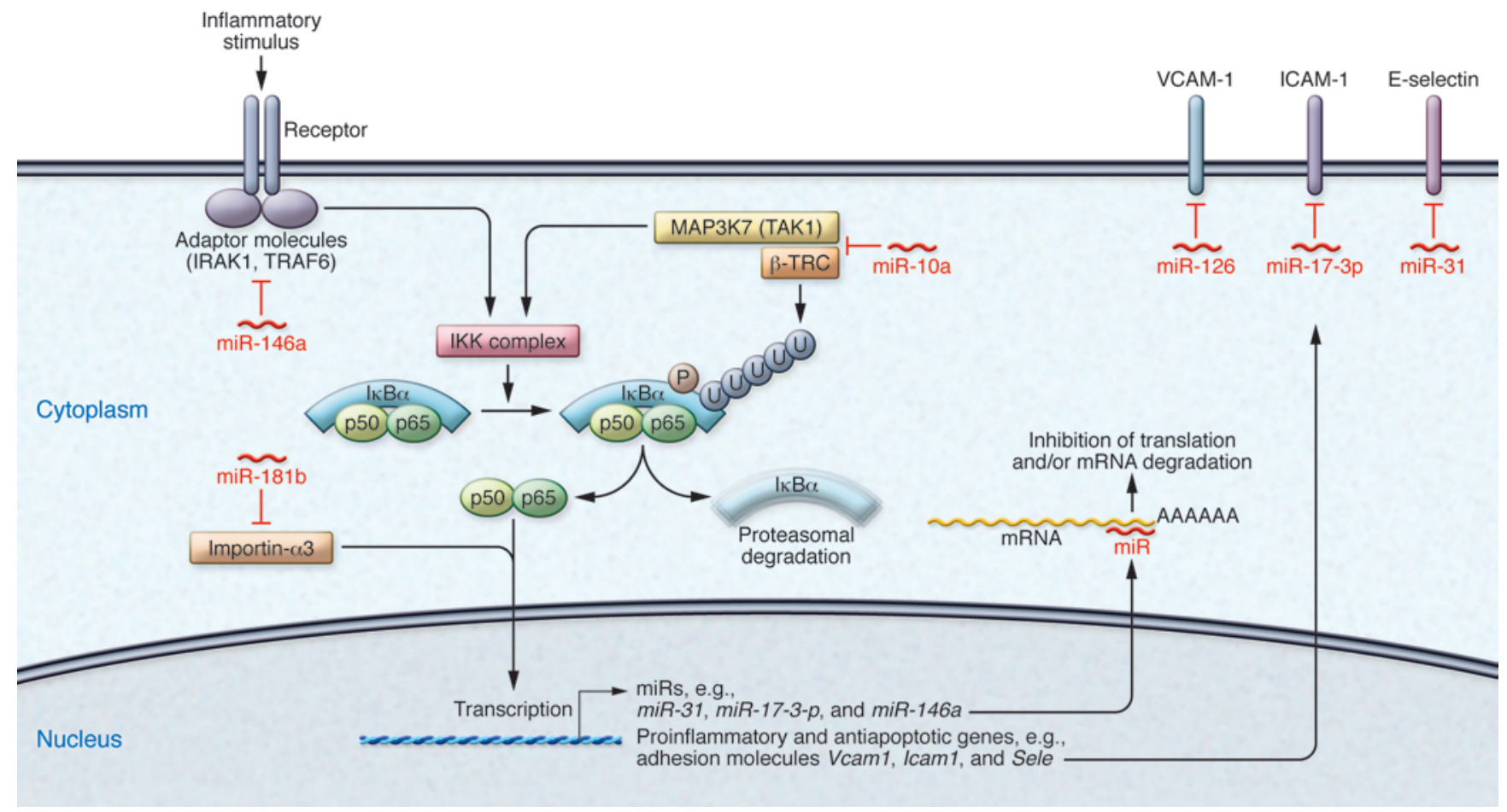

Figure 1

MicroRNA-based regulation of NF-KB signaling and endothelial activation. Multiple inflammatory stimuli lead to activation of the IKK complex, which phosphorylates $1 \kappa B$ and promotes its polyubiquitination and proteasomal degradation. NF-kB proteins (such as p50/p65 heterodimers) that are released from $\mathrm{I} \mathrm{KB}$ are imported into the nucleus via their nuclear localization signals, where they activate the transcription of proinflammatory genes, including vascular adhesion molecules (i.e., Vcam1, Icam1, Sele). Nuclear import of NF-кB is facilitated by importin proteins, including importin- $\alpha 3$. MicroRNAs bind to the $3^{\prime}$ UTRs of target mRNAs and inhibit their stability and/or translation. miR-181b elicits an antiinflammatory effect in endothelial cells by repressing importin- $\alpha 3$ expression, thereby inhibiting nuclear import of NF- $\kappa$ B. In addition, an endothelial-specific microRNA, miR-126, inhibits the expression of VCAM-1, and inflammation induces the expression of several microRNAs, including miR-31, miR-17-3p, and miR-146a, which participate in negative feedback loops. miR-31 negatively regulates E-selectin, miR-17-3p represses ICAM-1 expression, and miR-146a targets adaptor molecules (i.e., IRAK1, TRAF6) that are involved in inflammatory signal transduction. miR-10a, which is reduced in regions of blood vessels that are exposed to disturbed flow, represses MAP3K7 (TAK1) and $\beta$-TRC, which promote IKB degradation.

they associate preferentially with different

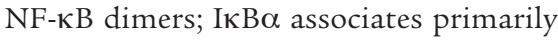
with the p50/p65 heterodimer. The binding of an IKB masks the classical nuclear localization sequence (NLS) of each NF-кB subunit and inhibits import to the nucleus.

Diverse stimuli, including ligand binding to proinflammatory cytokine receptors, TLR, and other receptors, such as RAGE, lead to activation of IKB kinases and NF- $\mathrm{KB}$ signaling via the canonical pathway (5). In this signaling cascade, IкB is phosphorylated on 2 conserved serines (S-32 and S-36 on IкB $\alpha$ ), polyubiquitinated, and targeted for degradation by the $26 \mathrm{~S}$ proteasome. This unmasks the NLS of NF- $\kappa \mathrm{B}$ subunits and enables transport to the nucleus. Nuclear transport of proteins with a classical NLS is mediated by a heterodimeric complex made up of proteins from the importin- $\alpha$ and importin- $\beta$ families, where the importin- $\alpha$ binds to the protein cargo and the importin- $\beta$ mediates interaction with the nuclear pore complex (6). When the complex reaches the nucleus, it binds RanGTP, which induces a conformational change that dissociates it. Of the 6 human importin- $\alpha$ isoforms, only $\alpha 3$ and $\alpha 4$ are capable of binding the NLS of p50 and p65 and mediating nuclear import (7). In the nucleus, $\mathrm{p} 50 / \mathrm{p} 65$ heterodimers recognize specific nucleotide sequences and transactivate gene expression through interactions with cofactors, other transcription factors, and histone acetyltransferases (5).

$\mathrm{NF}-\kappa \mathrm{B}$ signaling is autoregulated, since activation of this pathway induces the expression of a number of proteins that function as inhibitors of NF- $\mathrm{KB}$ activation. This includes rapid upregulation of $\mathrm{I} \kappa \mathrm{B} \alpha$ expression and replenishment of its cytoplasmic pool. Unbound I $\mathrm{B} \alpha$ contains an exposed NLS and is imported into the nucleus, where it associates with

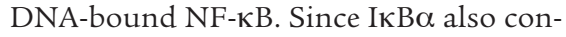
tains a nuclear export sequence, it binds exportins that transport the NF- $\kappa \mathrm{B} / \mathrm{I} \kappa \mathrm{B} \alpha$ complex back to the cytoplasm (5). Thus, the NF- $\kappa$ B pathway is tightly regulated to control the intensity and duration of an inflammatory response.

\section{MicroRNAs, endothelial cell activation, and NF-кB}

MicroRNAs are small noncoding RNAs ( 21 nucleotides in length) that bind primarily to the 3' UTR of mRNAs and decrease their stability and/or inhibit translation (8). Recently, several microRNAs have been implicated in the regulation of the inflammatory response in endothelial cells (Figure 1). For example, miR-126, an endothelial-specific microRNA (9), inhibits the expression of VCAM-1 and antagonizes the binding of leukocytes to endothelial cells in vitro (10). Addition- 
ally, miR-31 and miR-17-3p are induced following exposure of endothelial cells to TNF- $\alpha$, and these microRNAs negatively regulate E-selectin and ICAM-1 expression, respectively, thus acting in a negative feedback loop to restrain endothelial cell activation (11). miR-146a is another of a microRNA that participates in a negative feedback loop that tempers inflammatory signaling. In monocytes, miR-146a is transcriptionally induced via NF- $\mathrm{BB}$ binding to its promoter following activation of TLR signaling, and it represses upstream components of the TLR pathway, including IRAK1 and TRAF6 (12). The involvement of this microRNA in endothelial cell activation has not been addressed.

Disturbed blood flow promotes inflammation and atherosclerosis, in part by priming of canonical NF- $\mathrm{KB}$ signaling (13). Interestingly, miR-10a expression is lower in regions of the aorta that are exposed to disturbed flow (14). This microRNA regulates the expression of mitogen-activated kinase kinase kinase 7 (MAP3K7;TAK1) and $\beta$-transducin repeat-containing gene $(\beta$-TRC), both of which facilitate IкB $\alpha$ degradation. Inhibition of $\mathrm{miR}-10 \mathrm{a}$ therefore enhances NF-KB-dependent adhesion molecule expression in endothelial cells (14).

\section{miR-181b tempers endothelial activation}

The findings of Sun et al. (4) provide an additional example of the intersection between microRNAs and the NF- $\kappa \mathrm{B}$ pathway in endothelial cells. The authors elegantly demonstrate that miR-181b plays a key role in controlling endothelial activation. This is mediated by the regulation of importin- $\alpha 3$, a protein that facilitates the nuclear import of p50/p65. Using miR$181 \mathrm{~b}$ mimetics in vivo, they show promising protective effects in a murine model of sepsis that are consistent with a previous study in which endothelial cells expressed a dominant negative $\mathrm{I} \kappa \mathrm{B} \alpha(15)$. However, it remains to be seen whether over-expression of miR-181b can be sustained to provide protection against chronic inflammation. This is particularly relevant for chronic inflammatory vascular diseases such as atherosclerosis. Sun et al. additionally observed reduced levels of circulating miR$181 \mathrm{~b}$ in patients with sepsis (4). Their data provide a rationale for targeting endothelial cell NF- $\kappa \mathrm{B}$ signal transduction in septic patients. However, for this approach to be practical in patients, therapy would have to be effective when administered after the onset of sepsis. Whether miR-181b mimetics will be effective at later stages of sepsis is not addressed in the current body of work, and experiments in mouse and nonhuman primate models will be required before this treatment can be attempted in human clinical trials.

\section{MicroRNA-based diagnostics and therapeutics}

It is now apparent that several microRNAbased regulatory networks converge on NF-кB-dependent inflammatory pathways (refs. 4, 10, 11, 14, and Figure 1) and may contribute to diseases of the vasculature. Therapeutic manipulation of microRNA function is therefore being explored. Additionally, microRNAs can be detected in the circulation, and their expression is altered in disease conditions such as myocardial infarction (16) and septic shock (4). Therefore, microRNA expression may be utilized as a biomarker. Antisense microRNA inhibitors (anti-miRs) provide potent and long-term antagonism of microRNA function following intravenous or subcutaneous injection in animal models. For example, antagonism of miR-33a and miR$33 \mathrm{~b}-$ which repress a network of genes involved in fatty acid oxidation and synthesis as well as the cholesterol transporter ABCA1 - enhances the level of HDL while suppressing VLDL levels in nonhuman primate models (17).

The first clinical trial using an anti-miR approach is currently underway, targeting miR-122 (18), which was previously shown to be involved in HCV replication (19).Santaris Pharma A/S is now performing phase II clinical trials utilizing miR122 anti-miRs in HCV patients, and this approach appears to provide prolonged antiviral activity (18). Examples of therapeutic microRNA overexpression using microRNA mimetics are less common, but these approaches have shown some benefit in animal models (20). As opposed to antimiRs, which can be administered globally to target cell-restricted microRNAs, such as miR-122 (liver) or miR-126 (endothelium), microRNA mimetics require cell- or tissue-specific delivery to reduce unintended side effects of microRNA overexpression in nontarget cells. This remains a major technical hurdle for these approaches.

Interestingly, Sun et al. (4) show that intravenous delivery of miR-181b mimetics results in overexpression of miR-181b in the intimal layer of blood vessels (i.e., endothelium), but not in the media and adven- titia. Further experiments will be required to fully appreciate the cell and tissue distribution of injected miR-181b mimetics and the consequences of miR-181b overexpression in nonendothelial cells. It is intriguing that the effects of miR-181b treatment appear to be restricted to endothelial cells, since no effect on NF-кB-dependent gene expression was observed in peripheral blood mononuclear cells from miR-181binjected mice. The molecular mechanisms for this endothelial specificity are not clear at present, since importin- $\alpha 3$ is presumably expressed in many cell types.

Targeting miR-181b to control endothelial activation appears to be a very promising therapeutic approach, and subsequent work will define whether this microRNA is able to effectively tame inflammation in the setting of human vascular disease and/ or sepsis.

\section{Acknowledgments}

Research in the laboratories of J.E. Fish and M.I. Cybulsky is supported by operating grants from the Heart and Stroke Foundation of Ontario and the Canadian Institutes of Health Research.

Address correspondence to: Jason E. Fish or Myron I. Cybulsky, Toronto General Research Institute, University Health Network, MaRS Centre, Toronto Medical Discovery Tower, 101 College Street, Toronto, Ontario, M5G 1L7, Canada. Phone: 416.581.7495; Fax: 416.581.7484; E-mail: jason.fish@utoronto. ca (J.E. Fish). Phone: 416.581.7483; Fax: 416.581.7484; E-mail: myron.cybulsky@ utoronto.ca (M.I. Cybulsky).

1. Pober JS, Sessa WC. Evolving functions of endothelial cells in inflammation. Nat Rev Immunol. 2007;7(10):803-815.

2. Gareus R, et al. Endothelial cell-specific NF-kap$\mathrm{paB}$ inhibition protects mice from atherosclerosis. Cell Metab. 2008;8(5):372-383.

3. Kanters E, et al. Inhibition of NF-kappaB activation in macrophages increases atherosclerosis in LDL receptor-deficient mice. J Clin Invest. 2003; 112(8):1176-1185.

4. Sun X, et al. MicroRNA-181b regulates NF-кBmediated vascular inflammation. J Clin Invest. 2012;122(6):1973-1990.

5. Perkins ND. Integrating cell-signalling pathways with NF-kappaB and IKK function. Nat Rev Mol Cell Biol. 2007;8(1):49-62.

6. Lange A, Mills RE, Lange CJ, Stewart M, Devine SE, Corbett AH. Classical nuclear localization signals: definition, function, and interaction with importin alpha. J Biol Chem. 2007;282(8):5101-5105.

7. Fagerlund R, Kinnunen L, Kohler M, Julkunen I, Melen K. NF-\{kappa\}B is transported into the nucleus by importin $\{$ alpha\} 3 and importin \{alpha\}4.J Biol Chem. 2005;280(16):15942-15951.

8. Bartel DP. MicroRNAs: target recognition and regulatory functions. Cell. 2009;136(2):215-233. 
9. Fish JE, et al. miR-126 regulates angiogenic signaling and vascular integrity. Dev Cell. 2008;15(2):272-284.

10. Harris TA, Yamakuchi M, Ferlito M, Mendell JT, Lowenstein CJ. MicroRNA-126 regulates endothelial expression of vascular cell adhesion molecule 1. Proc Natl Acad Sci U S A. 2008;105(5):1516-1521.

11. Suarez Y, Wang C, Manes TD, Pober JS. Cutting edge: TNF-induced microRNAs regulate TNF-induced expression of E-selectin and intercellular adhesion molecule- 1 on human endothelial cells: feedback control of inflammation. J Immunol. 2010;184(1):21-25.

12. Taganov KD, Boldin MP, Chang KJ, Baltimore D NF-kappaB-dependent induction of microRNA miR-146, an inhibitor targeted to signaling proteins of innate immune responses. Proc Natl Acad Sci US A. 2006;103(33):12481-12486.
13. Hajra L, Evans AI, Chen M, Hyduk SJ, Collins T, Cybulsky MI. The NF-kappa B signal transduction pathway in aortic endothelial cells is primed for activation in regions predisposed to atherosclerotic lesion formation. Proc Natl Acad Sci U S A. 2000; 97(16):9052-9057.

14. Fang Y, Shi C, Manduchi E, Civelek M, Davies PF. MicroRNA-10a regulation of proinflammatory phenotype in athero-susceptible endothelium in vivo and in vitro. Proc Natl Acad Sci U S A. 2010; 107(30):13450-13455.

15. Ye X, Ding J, Zhou X, Chen G, Liu SF. Divergent roles of endothelial NF-kappaB in multiple organ injury and bacterial clearance in mouse models of sepsis. J Exp Med. 2008;205(6):1303-1315.

16. Wang GK, et al. Circulating microRNA: a novel potential biomarker for early diagnosis of acute myocardial infarction in humans. Eur Heart J. 2010; 31(6):659-666.

17. Rayner KJ, et al. Inhibition of miR-33a/b in nonhuman primates raises plasma HDL and lowers VLDL triglycerides. Nature. 2011;478(7369):404-407.

18. van Rooij E, Purcell AL, Levin AA. Developing microRNA therapeutics. Circ Res. 2012;110(3):496-507.

19. Jopling CL, Yi M, Lancaster AM, Lemon SM, Sarnow P. Modulation of hepatitis C virus RNA abundance by a liver-specific MicroRNA. Science. 2005; 309(5740):1577-1581

20. Trang P, et al. Systemic delivery of tumor suppressor microRNA mimics using a neutral lipid emulsion inhibits lung tumors in mice. Mol Ther. 2011; 19(6):1116-1122.

\title{
Getting to the finish line with mTORC1-targeted therapy
}

\author{
Elizabeth Henske
}

Brigham and Women's Hospital, Boston, Massachusetts, USA.

\begin{abstract}
The mammalian target of rapamycin (mTOR) pathway is activated in the majority of human malignancies and thus seems a likely therapeutic target. However, this pathway is genetically complex, complicating studies using pharmacologic mTOR inhibitors. In this issue of the JCI, Hoshii et al. examined mice deficient in one of the mTOR effector complex proteins, Raptor, to elucidate the role of mTORC1 in leukemia. They convincingly demonstrate that Raptor deficiency, with consequent mTORC1 inhibition, blocks differentiation of leukemia cells and prolongs survival, but also allows a population of leukemia-initiating cells to persist in what appears to be a state of dormancy. Translating this new understanding into effective therapeutic strategies will require further study of the molecular mechanisms that underlie these processes.
\end{abstract}

\section{Introduction}

The majority of human cancers are characterized by activation of the serine/ threonine kinase mTOR through one or both of its complexes. Given this frequency, the pathway seems a promising therapeutic target, and as of April 2012, ClinicalTrials.gov listed more than 100 cancer clinical trials of mTOR inhibitors, most of which use an mTOR inhibitor in combination with a conventional chemotherapeutic agent and/or another targeted agent. However, it has been challenging to deduce how best to target mTOR therapeutically in human malignancy. Even in benign tumors in tuberous sclerosis complex (TSC) patients (1), in which mTOR activation is generally believed to be the

Conflict of interest: The author has declared that no conflict of interest exists.

Citation for this article: J Clin Invest. 2012; 122(6):1970-1972. doi:10.1172/JCI64227. primary driver of tumorigenesis, treatment with mTOR inhibitors yields only partial tumor regression, with regrowth when the agents are stopped (2-4).

Targeting mTOR in human cancer is complex for many reasons. The two distinct complexes in which mTOR participates have different functions, different sensitivity to mTOR inhibition, and can indirectly regulate each other $(5,6)$. These two complexes, termed mTOR complex 1 (mTORC1) and mTORC2 are distinguished in part by the mTOR-binding partners, Raptor and Rictor, respectively (Figure 1). The regulation of these complexes, their cellular functions, and their kinase targets are non-overlapping. mTORC1 controls protein translation, cell size, and autophagy through multiple substrates, which include p70 ribosomal protein S6 kinase, eukaryote translation initiation factor $4 \mathrm{E}$ binding protein, and ULK1 (7-10). mTORC1 is regulated in part by the Ras homolog Rheb, which is inhibited by the TSC1 and TSC2 proteins. mTORC2 appears to have completely different upstream regulators and downstream kinase targets, which include Akt $(5,11)$. For example, the TSC proteins, which act via Rheb to inhibit mTORC1, do not directly inhibit mTORC2. Finally, targeting mTOR in cancer can lead to undesired effects on proliferation and survival. For example, feedback loops induced by mTORC1 inhibition can activate kinases such as Akt that drive proliferation, and mTORC1 downregulation induces autophagy, thereby promoting cell survival in some tumors (12-14).

\section{The power of genetics}

Although mTOR kinase is named for its inhibitory compound (rapamycin), rapamycin and similar agents (often called rapalogs) inhibit only mTORC1, and their inhibition of mTORC1 is incomplete. This is not surprising, since rapamycin actually inhibits mTOR indirectly by binding to FKBP12. Thus mTOR's other name, FK506 binding protein 12-rapamycin associated protein (FRAP), is perhaps a more accurate designation. Further complexity is introduced by the fact that rapalogs can affect the activity of mTORC 2 in some cell types, particularly at higher doses, and because the effects of rapalogs on specific substrates are dynamically regulated. For example, inhibition of S6K phosphorylation is often sustained for longer periods than inhibition of 4EBP1 phosphorylation. 